Geophysical and Astrophysical Fluid Dynamics

Vol. 00, No. 00, 00 Month 2017, 1-20

\title{
Stability of filaments of uniform quasi-geostrophic potential vorticity
}

\author{
JEAN N. REINAUD* \\ Mathematical Institute, North Haugh, St Andrews, KY169SS, UK \\ (Received 00 Month 20xx; final version received 00 Month 20xx)
}

\begin{abstract}
We analyse the linear stability of filaments of uniform potential vorticity with a horizontal axis in a quasigeostrophic flow. For a single filament, the situation corresponds to the simplest three-dimensional shear zone in a rapidly rotating, continuously stably stratified fluid. Yet, this has not been formally addressed to our knowledge. We show that the filament is sensitive to the Kelvin-Helmholtz instability for perturbations in a finite range of streamwise wavenumbers $0<k<k_{c}$, similarly to the classical situation of a twodimensional strip of uniform vorticity. We also analyse the stability of a jet formed by two parallel filaments of opposite PV whose axes are located on the same horizontal plane as well as the stability of 'hetonic' filaments. Hetonic filaments consist of a pair of opposite PV filaments located at different heights. These can be sensitive to baroclinic instabilities over a wide range of longitudinal wavenumbers.
\end{abstract}

Keywords: Vortex dynamics; Quasi-geostrophy

\section{Introduction}

Medium to large scale oceanic and atmospheric flows are strongly influenced by the planetary rotation and the stable density stratification of the fluid. In these environments, shear zones are ubiquitous, and their destabilisation are paramount to the formation of oceanic eddies and weather patterns. We consider the simplest configuration leading to a shear zone in a three-dimensional rapidly rotating, continuously stratified flow, namely a filament of uniform potential vorticity anomaly with a horizontal axis. In practice, these filaments are also observed during the destabilisation of unstable vortices (see Dritschel et al. 2005) during the strong interaction between like-signed vortices (see e.g. Reinaud and Dritschel 2005, and Bambrey et al. 2007) during the breaking of unstable hetons (see Reinaud and Carton 2009) as well as in geostrophic turbulence (see McWilliams 1990, Reinaud et al. 2003).

Shear zones associated with vorticity filaments are generic to nearly all high Reynolds flow regimes. First, Helmholtz (1868) showed that a vortex sheet in a two-dimensional flow was unstable. Then, the linear stability of a two-dimensional strip of uniform vorticity $w_{0}$ and width $d$ was first addressed by Lord Rayleigh (see, for example, Rayleigh 1896, §368). In that case, the growth rate $\sigma_{i}(k)$ of a perturbation of longitudinal wavenumber $k$ is given by $\sigma_{i}^{2}=\left(\omega_{0} / 2\right)^{2}\left[\mathrm{e}^{-2 k d}-(1-k d)^{2}\right]$ which has a maximum for $k d \simeq 0.797$ corresponding to a wavelength $\lambda \simeq 7.89 d$. Similar results were obtained for continuous vorticity distributions (see, for example, Michalke 1964, for a hyperbolic tangent velocity profile). These results were extended to many other applications (e.g. compressible or reacting mixing layers). The nonlinear evolution of stratified two-dimensional mixing layers at infinite Froude number where gravitational effects are negligible compared to inertial ones have been studied in Reinaud et al. (2000), Fontane et al. (2008).

Stably stratified mixing layers at finite Froude number received also extensive attention both in two-dimensions (see, e.g., Staquet 1995) and in three-dimension (see, e.g., Mashayek

*Corresponding author: Email: jean.reinaud@st-andrews.ac.uk 
and Peltier 2013) due to the role of the destabilisation of the shear layers to the transition to turbulence.

In geophysical applications, Waugh and Dritschel (1991) consider the stability of a strip of potential vorticity in a two-dimensional shallow water model and other geophysically motivated situations, while Dritschel and Polvani (1992) considered the stability of the strip of vorticity on a sphere. On the other hand, Juckes (1995) studied the stability of a strip of uniform buoyancy in surface quasi-geostrophy (SQG), where buoyancy plays a role similar to vorticity. The stability of a distributed strip of buoyancy was addressed in Reinaud et al. (2016). Using continuous buoyancy distribution avoids the unphysical infinite longitudinal velocity observed at the edges of the uniform buoyancy strip in SQG.

In a three-dimensional, stably stratified and rotating fluid at finite Rossby and Froude number, Dritschel and Viúdez (2003) considered, for the first time, a filament of distributed potential vorticity. But the authors did not address the linear stability of the filament, but focused on the vertical velocities induced during the destabiilsation of the filament.

In the present paper we address the linear stability of a single filament of uniform quasigeostrophic potential vorticity. The quasi-geostrophic model is the simplest dynamical model which takes into account the dominant effects of the background planetary rotation and of the stable density stratification observed for medium to large scales in the atmosphere and the oceans. We show, as expected, that the filament is sensitive to a Kelvin-Helmholtz type instability for perturbations in a finite range of longitudinal wavenumbers. We also consider the nonlinear evolution of the filament when excited by various initial perturbations. The formalism derived to study the linear stability of the single filament can be straightforwardly adapted to multiple, parallel filaments. We next consider two simple configurations with two parallel filaments of uniform PV of opposite sign. In the first case the axes of the filaments lie on the same horizontal plane, forming a jet. We show that the jet is sensitive to two modes of instability (the sinuous and the varicose modes) with the sinuous mode being the most amplified. In the second, case the two filaments are on the top of one another, and the flow has a baroclinic component similar to hetons. In this case the configuration is unstable to a perturbations in a very large range of longitudinal wavenumbers. The instability for the highest wavenumbers is initially restricted to a thin region near where the filaments touch. We also include the analysis of intermediate cases where the filaments are still touching but whose axes are offset both horizontally and vertically.

The paper is organised as follows. The basic equations are given in section 2 . The linear stability of a single filament is derived in section 3 . The nonlinear evolution of a single filament is discussed in section 4. Results for jets and hetonic filaments are presented in sections 5 and 6 respectively. Conclusions are given in section 7 .

\section{The quasi-geostrophic model}

We consider an inviscid fluid in a uniformly rotating and continuously stratified fluid in an adiabatic evolution under the Boussinesq approximation. We make use of the quasi-geostrophic (QG) model which is the simplest dynamical model capturing the leading effects of the background planetary rotation and the density stratification of the fluid. In the present context, the QG model derives from an asymptotic expansion of Euler's equations in term of Rossby number for Burger number $B u=\mathrm{O}(1)$. More specifically, the QG model is valid provided $F r^{2} \ll R o \ll 1$, where $F r=U /(N H)$ is the Froude number and $R o=U /(f L)$ is the Rossby number. Here, $U$ is a characteristic scale of horizontal velocity, $L$ and $H$ are characteristic horizontal and vertical length scales respectively. The Coriolis frequency is denoted $f$ and corresponds to the projection onto the local vertical direction of twice the planetary rotation. The buoyancy or Brunt-Väissälä frequency is denoted $N$ and is the oscillation frequency of 
the fluid parcel displaced vertically in the stably stratified fluid. For simplicity we assume $f$ and $N$ constant. The Burger number can be written as a function of the Rossby and Froude number $B u=(R o / F r)^{2}$. In the QG model, all fields can be deduced from the distribution of potential vorticity anomaly $(\mathrm{PV}) q$. The governing equations read

$$
\frac{\partial q}{\partial t}+J(\varphi, q)=0
$$

$J(f, g)=(\partial f / \partial x)(\partial g / \partial y)-(\partial f / \partial y)(\partial g / \partial x)$ is the Jacobian. PV is defined as

$$
q=\frac{\partial^{2} \varphi}{\partial x^{2}}+\frac{\partial^{2} \varphi}{\partial y^{2}}+\frac{\partial^{2} \varphi}{\partial z^{2}}
$$

where the physical vertical coordinate $z_{p}$ is replaced by the stretched coordinate $z=z_{p} N / f$. $\mathrm{PV}$ is materially conserved as stated by (1). Equation (2) can be formally inverted to obtain

$$
\varphi(\boldsymbol{x})=-\frac{1}{4 \pi} \iiint \frac{q\left(\boldsymbol{x}^{\prime}\right)}{\left|\boldsymbol{x}-\boldsymbol{x}^{\prime}\right|} \mathrm{d}^{3} \boldsymbol{x},
$$

from wihich the velocity field derives

$$
(u, v)=\left(-\frac{\partial}{\partial y}, \frac{\partial}{\partial x}\right) \varphi=\frac{1}{4 \pi} \int\left(\sum_{\text {contours at height z' }} \oint_{C} \frac{\Delta Q_{C}}{\left|\boldsymbol{x}-\boldsymbol{x}^{\prime}\right|}\left(\mathrm{d} x^{\prime}, \mathrm{d} y^{\prime}\right)\right) \mathrm{d} z^{\prime},
$$

where Green's theorem is used to convert the horizontal surface integrals into contour integrals. $\Delta Q_{C}$ is the PV jump across contour $C$.

\section{Linear stability of a single filament}

\subsection{Basic state}

We consider as a basic state a horizontal cylindrical filament of radius $a$ and of uniform PV $Q_{0}$ whose axis is the $x$-axis. The PV field is defined by

$$
\bar{q}(R)= \begin{cases}Q_{0}, & R \leq a \\ 0, & R>a\end{cases}
$$

where $R=y^{2}+z^{2}$. We also define the polar angle $\theta=\arctan (z / y)$. By symmetry, the streamfunction for the basic state $\bar{\varphi}=\bar{\varphi}(R)$. For $R \leq a$ we have

$$
\Delta \bar{\varphi}(R)=\frac{1}{R} \frac{\mathrm{d}}{\mathrm{d} R}\left(R \frac{\mathrm{d} \bar{\varphi}}{\mathrm{d} R}\right)=Q_{0}
$$

which leads to

$$
\frac{\mathrm{d} \bar{\varphi}}{\mathrm{d} R}=\frac{Q_{0} R}{2}+\frac{A}{R} .
$$

We have $A=0$ for regularity along the $x$-axis. By integration we obtain

$$
\bar{\varphi}=\frac{Q_{0} R^{2}}{4},
$$




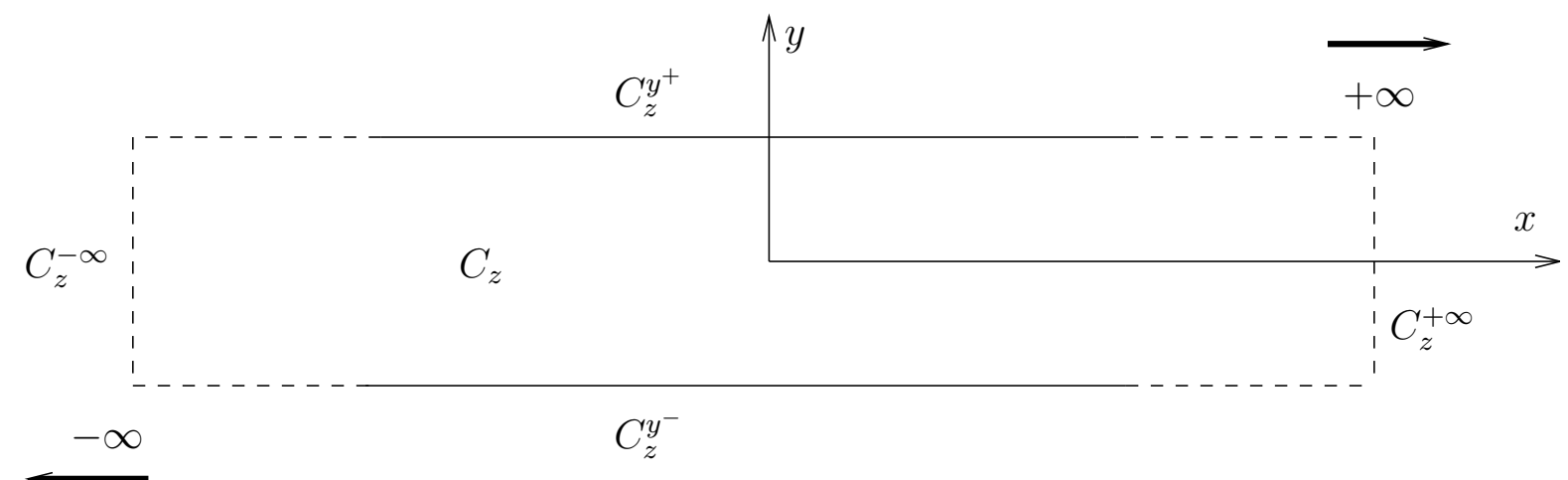

Figure 1. Linear stability analysis: geometry of the infinite contour bounding the infinite filament at height $z$.

leading to the geostrophic horizontal velocities and the buoyancy field for the basic state

$$
\begin{aligned}
& \bar{u}=-\frac{\partial \bar{\varphi}}{\partial y}=-\frac{\mathrm{d} \bar{\varphi}}{\mathrm{d} R} \frac{y}{R}=-\frac{Q_{0}}{2} y, \\
& \bar{v}=\frac{\partial \bar{\varphi}}{\partial x}=0, \\
& \bar{b}=N \frac{\partial \bar{\varphi}}{\partial z}=\frac{N Q_{0}}{2} z .
\end{aligned}
$$

The external solution, for $R>a$ corresponds to

$$
\Delta \bar{\varphi}(R)=0 \quad \Longrightarrow \quad \bar{\varphi}=B \ln R+D .
$$

Matching both solutions for $\bar{\varphi}$ and $\mathrm{d} \bar{\varphi} / \mathrm{d} R$ at $R=a$, we obtain $B=Q_{0} a^{2} / 2$ and $D=$ $Q_{0} a^{2}(1-2 \ln a) / 4$. We however do not need this part of the solution in our study. It should be noted that the solution for the basic state $(b / N, v)$ is mathematically similar to the solution $(u, v)$ of the two-dimensional Rankine vortex.

\subsection{Linear stability}

We next address the linear stability of a single filament of uniform PV, centred along the $x$-axis. We consider horizontal perturbations of the filament's boundary. We do not consider vertical perturbations as there is no vertical advection in $\mathrm{QG}$, hence the geostrophic velocity cannot displace the filament in this direction. At each height $z$ through the filament, the filament's boundary is a single infinite 'closed' contour $C_{z}=C_{z}^{y^{+}} \cup C_{z}^{+\infty} \cup C_{z}^{y^{-}} \cup C_{z}^{-\infty}$, where $C_{z}^{y^{+}}$and $C_{z}^{y^{-}}$are infinite lines along the (perturbed) filament, and $C_{z}^{+\infty}$ and $C_{z}^{-\infty}$ are lines crossing the filament (at $x \rightarrow \pm \infty$ ), see figure 1 .

The filament's longitudinal boundaries $y^{+}($for $y>0)$ and $y^{-}($for $y<0)$ at height $z$ are perturbed

$$
y^{ \pm}=\bar{y}^{ \pm}+\eta^{ \pm} \mathrm{e}^{\mathrm{i}(k x-\sigma t)}
$$

where $\bar{y}^{ \pm}$is the $y$-coordinate of the unperturbed filament, satisfying $\left(\bar{y}^{ \pm}\right)^{2}+z^{2}=R^{2}, k$ is the longitudinal wavenumber of the perturbation, and $\sigma=\sigma_{r}+\mathrm{i} \sigma_{i}$, where $\sigma_{r}$ is the mode's frequency and $\sigma_{i}$ is its growth rate. We next note that the transversal velocity $v=\bar{v}+v^{\prime}=v^{\prime}$ reduces to the perturbation velocity $v^{\prime}$ since the basic state transversal velocity $\bar{v}=0$. We linearise the transversal displacement

$$
\frac{\mathrm{D} y^{ \pm}}{\mathrm{D} t}=v=\int_{-a}^{a} \frac{\Delta q_{C_{z^{\prime}}}}{4 \pi} \oint_{C_{z^{\prime}}} \frac{\mathrm{d} y^{\prime}}{\sqrt{\left(x-x^{\prime}\right)^{2}+\left(y^{ \pm}-y^{\prime}\right)^{2}+\left(z-z^{\prime}\right)^{2}}} \mathrm{~d} z^{\prime},
$$


using $\mathrm{d} y^{\prime}=\mathrm{d}\left(\bar{y}^{\prime \pm}+\eta^{\prime \pm} \mathrm{e}^{\mathrm{i}\left(k x^{\prime}-\sigma t\right)}\right)=\mathrm{i} k \eta^{\prime \pm} \mathrm{e}^{\mathrm{i}\left(k x^{\prime}-\sigma t\right)} \mathrm{d} x^{\prime}$ along $C_{z}^{y^{+}}$and $C_{z}^{y^{-}}$respectively and using the fact that the integrals along $C_{z}^{+\infty}$ and $C_{z}^{-\infty}$ cancel out each other, to obtain

$$
-\mathrm{i} \sigma \eta^{ \pm} \mathrm{e}^{\mathrm{i}(k x-\sigma t)}-\mathrm{i} k \frac{Q_{0}}{2} \bar{y}^{ \pm} \eta^{ \pm} \mathrm{e}^{\mathrm{i}(k x-\sigma t)}=\int_{-a}^{a} \frac{\Delta q_{C_{z^{\prime}}}}{4 \pi}\left(-G^{+}-G^{-}\right) \mathrm{d} z^{\prime},
$$

where

$$
\begin{aligned}
G^{+} & =\int_{-\infty}^{\infty} \frac{\mathrm{i} k \eta^{\prime+} \mathrm{e}^{\mathrm{i}\left(k x^{\prime}-\sigma t\right)}}{\sqrt{\left(x-x^{\prime}\right)^{2}+\left(\bar{y}^{ \pm}-\bar{y}^{\prime+}\right)^{2}+\left(z-z^{\prime}\right)^{2}}} \mathrm{~d} x^{\prime}, \\
G^{-} & =\int_{\infty}^{-\infty} \frac{\mathrm{i} k \eta^{\prime-} \mathrm{e}^{\mathrm{i}\left(k x^{\prime}-\sigma t\right)}}{\sqrt{\left(x-x^{\prime}\right)^{2}+\left(\bar{y}^{ \pm}-\bar{y}^{\prime}\right)^{2}+\left(z-z^{\prime}\right)^{2}}} \mathrm{~d} x^{\prime} .
\end{aligned}
$$

Substituting $u=x^{\prime}-x,\left(x^{\prime}=u+x\right)$, we obtain

$$
G^{+}=\mathrm{e}^{\mathrm{i}(k x-\sigma t)} \int_{-\infty}^{\infty} \frac{\mathrm{i} k \eta^{\prime} \mathrm{e}^{\mathrm{i} k u}}{\sqrt{u^{2}+\left(\bar{y}^{ \pm}-\bar{y}^{\prime+}\right)^{2}+\left(z-z^{\prime}\right)^{2}}} \mathrm{~d} u
$$

and a similar formula for $G^{-}$. Defining $\varrho=\sqrt{\left(\bar{y}^{ \pm}-\bar{y}^{\prime+}\right)^{2}+\left(z-z^{\prime}\right)^{2}}$. We then evaluate the integral directly using

$$
\int_{-\infty}^{\infty} \frac{\mathrm{e}^{\mathrm{i} k u}}{\sqrt{\varrho^{2}+u^{2}}} \mathrm{~d} u=2 \mathrm{~K}_{0}(k \varrho),
$$

from Abramowitz and Stegun (1972), p 376, formula 9.6.21, where $\mathrm{K}_{0}$ is the zeroth-order modified Bessel function of the second kind. At this stage, it is convenient to use the substitution $z^{\prime}=a \sin \theta^{\prime},-\pi / 2 \leq \theta^{\prime}<3 \pi / 2$, such that $\theta^{\prime} \in[-\pi / 2, \pi / 2)$ is used to represent the $y^{+}$-side of the filament, while $\theta^{\prime} \in[\pi / 2,3 \pi / 2)$ is used to represent the $y^{-}$-side of the filament. The change of sign in $\mathrm{d} z^{\prime}$ for $\theta^{\prime} \in[\pi / 2,3 \pi / 2)$ allows to swap the limits in $G^{-}$such that both $G^{+}$ and $G^{-}$have the same form, i.e. both are integrals from $-\infty$ to $+\infty$. Finally we reach the eigen-problem

$$
\sigma \eta(\theta)+k \frac{Q_{0}}{2} \bar{y}(\theta) \eta(\theta)=\int_{-\pi / 2}^{3 \pi / 2} \frac{\Delta q_{C_{z}}}{2 \pi} k \eta\left(\theta^{\prime}\right) \mathrm{K}_{0}(k \varrho) a \cos \theta^{\prime} \mathrm{d} \theta^{\prime} .
$$

after simplification by the common factor $-\mathrm{ie}^{\mathrm{i}(k x-\sigma t)}$. The integral in the rhs is evaluated numerically, by discretising the range of $\theta^{\prime}$ into $n_{i}$ equally spaced intervals. In each interval, the integration w.r.t $\theta^{\prime}$ is performed by 2-point Gaussian quadrature. This leads to a $n_{i} \times n_{i}$ eigenvalue problem. Convergence is checked by varying $n_{i}$ from 200 to 2000. Results are reported for the highest resolution, namely $n_{i}=2000$, for which convergence is confirmed.

Results for a single filament are presented in figure 2. We calculate the growth rates for $k a \in[0.001,1.1]$ with a increment of $\Delta(k a)=0.001$. It should be noted that $\sigma=0$ for $k=0$ from (18). The overall shape of the curve $\sigma_{i}=\sigma_{i}(k a)$ is similar to the one obtained in two-dimensions for the uniform vorticity strip. Instability occurs for $0<k a<(k a)_{c}$, where $(k a)_{c} \simeq 1.067$. This means that the growth rate of perturbations of infinite wavelength $(k a \rightarrow 0)$ vanishes, and that there is cut-off for the stability of short waves: short waves are stable. The maximum growth rate is $\sigma_{i}^{\max } \simeq 0.069$ for $k a^{\max } \simeq 0.676$ corresponding to a wavelength $\lambda^{\max } \simeq 4.65 d$, where $d=2 a$ is the diameter of the filament. This is significantly less than the wavelength of the most amplified mode for the two-dimensional vorticity strip. This is however close to the wavelength of the most amplified mode for the so-called 'elliptical' surface buoyancy distribution analysed in SQG by Reinaud et al. (2016), where $k a_{S Q G}^{\max }=0.729$. This is expected as, as the elliptical surface buoyancy strip is obtained by replacing a filament of PV by a singular sheet of PV of same vertically integrated PV (see Dritschel 2011 and Scott 2011). 


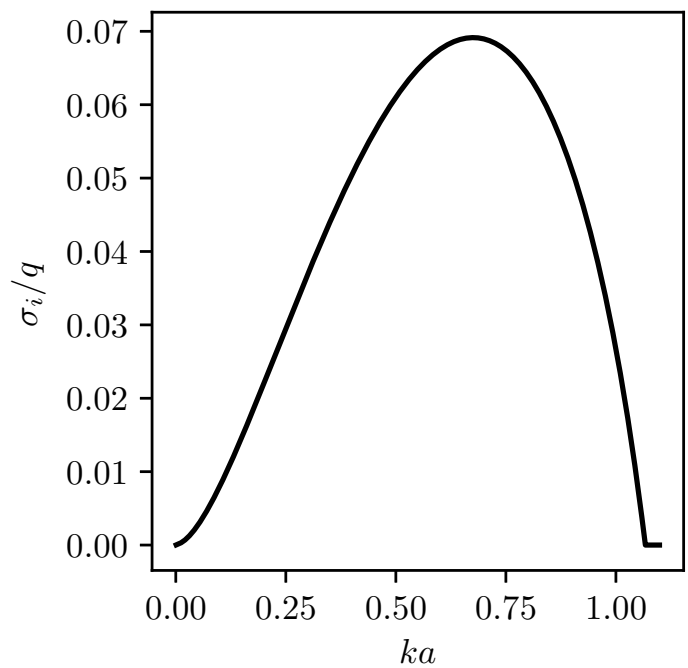

Figure 2. Growth rate $\sigma_{i}$ of the unstable mode for a single filament rescaled by the filament's PV $q$ vs the nondimensional longitudinal wave number $k a$.

\section{Nonlinear evolution of a single filament}

We next perform high resolution numerical simulations of the nonlinear evolution of a single filament of PV using CASL, the Contour Advective Semi-Lagrangian algorithm developed by Dritschel and Ambaum (1997). The computational domain is triply periodic, and of size $[2 \pi]^{3}$. The PV field is represented in a Lagrangian way by set of contours (of PV jumps) bounding regions of uniform PV. The velocity of the nodes discretising the contours is obtained on a Eulerian grid by inverting (2) in spectral space. For this the Lagrangian PV field is first converted to a fine gridded field of resolution $2048^{3}$ which is locally averaged onto a coarser inversion grid $512^{3}$, where the velocity field is obtained by inversion. The velocity of the nodes is then obtained by interpolation from the gridded values. Complexity of the contours is controlled by the Contour Surgery algorithm defined in Dritschel (1988) with standard set-up. Time integration is done with a standard fourth order Runge-Kutta algorithm with a time step $\Delta t=2 \pi /(20|q|)$.

In the first numerical experiment, the filament is initially perturbed by the most amplified mode. Since the most amplified mode has a wavelength of only $\lambda^{\max } \simeq 4.65 d$, we consider two periods in our computational box to avoid the strong influence of the neighbouring periodic boxes in the $y$ - and $z$-directions. This means that the filament's radius is set to $a=0.338$ and the filament's boundary is displaced by a wave $\epsilon \sin 2 x$, with $\epsilon=0.01$. Snapshots of the evolution are presented in figure 3. The amplitude of the perturbation grows and two billows are formed. Each billow is surrounded by a tongue of PV which is continuously stretched by the roll-up of the billows. Eventually the tongues merge. The tongues thin as they are stretched due to incompressibility and eventually break into small scale debris surrounding the billows.

To characterise the early growth of the perturbation, we extract the contour initially lying at $z=0$ and corresponding to $\bar{y}=-a$. For this contour, we determine $\bar{\eta}=\sqrt{(y-\bar{y})^{2} / n_{p}}$ as a function of time for $t \in[2,9.5]$, where the regime is nearly linear. Here $n_{p}$ is the number of nodes discretising the contour for $-\pi<x<\pi$. The shape of the contour as well as the evolution of $\bar{\eta}$ (scale per unit of PV) is presented in figure 4 . The slope of $\log \bar{\eta} / q \simeq 0.069$, the growth rate predicted by the linear stability analysis.

In the next experiment, we investigate further the growth of the billows by initially per- 

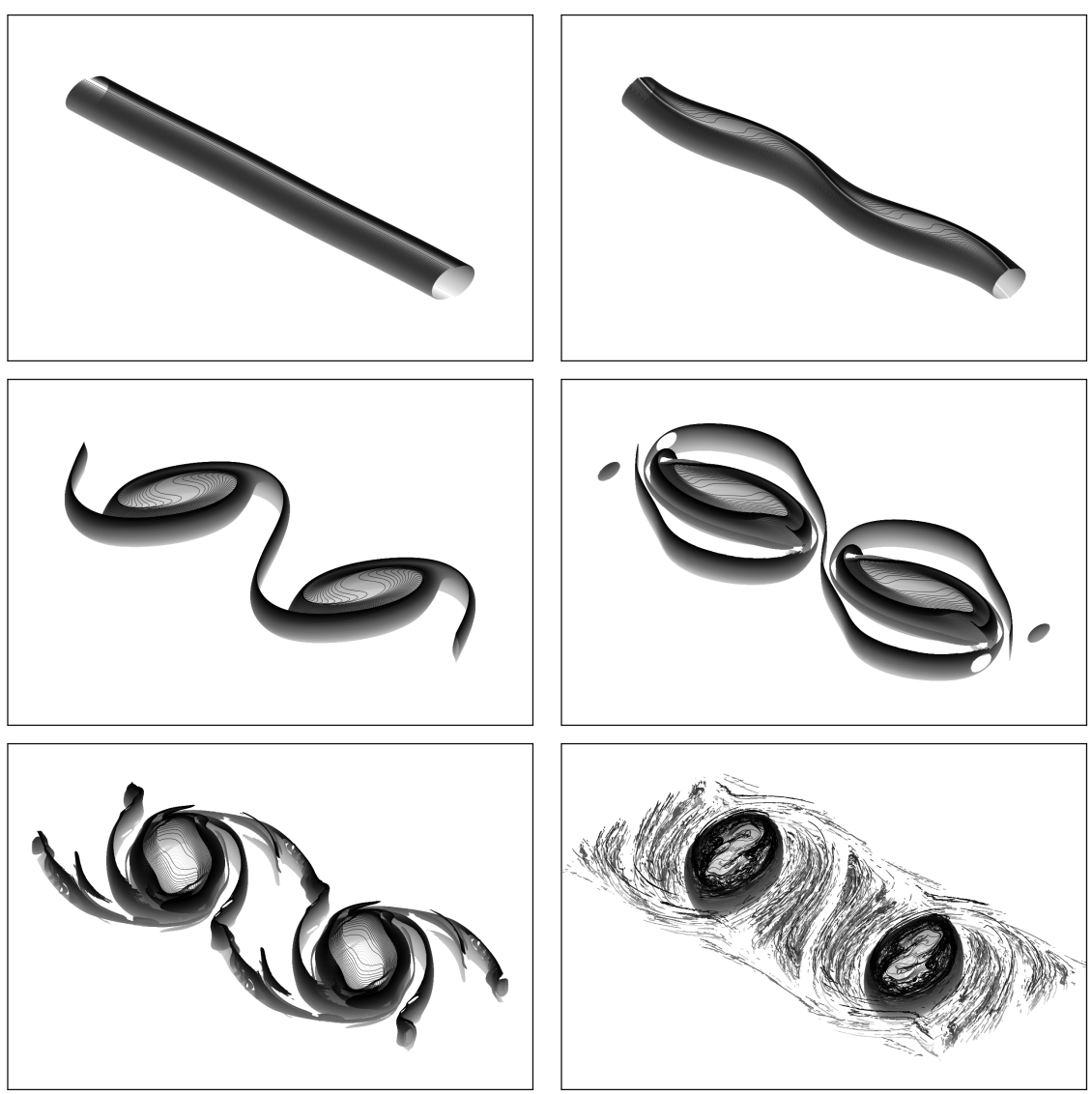

Figure 3. Evolution of a single filament of uniform PV $q=2 \pi$ of radius $a=0.338$, initially perturbed by the most amplified mode of nondimensional wavenumber $k a=0.676$ at $t=0,7,12.5,15.5,23$ and 90. Colour of the contours indicates the height of the contour, with darker contours are nearer the top.
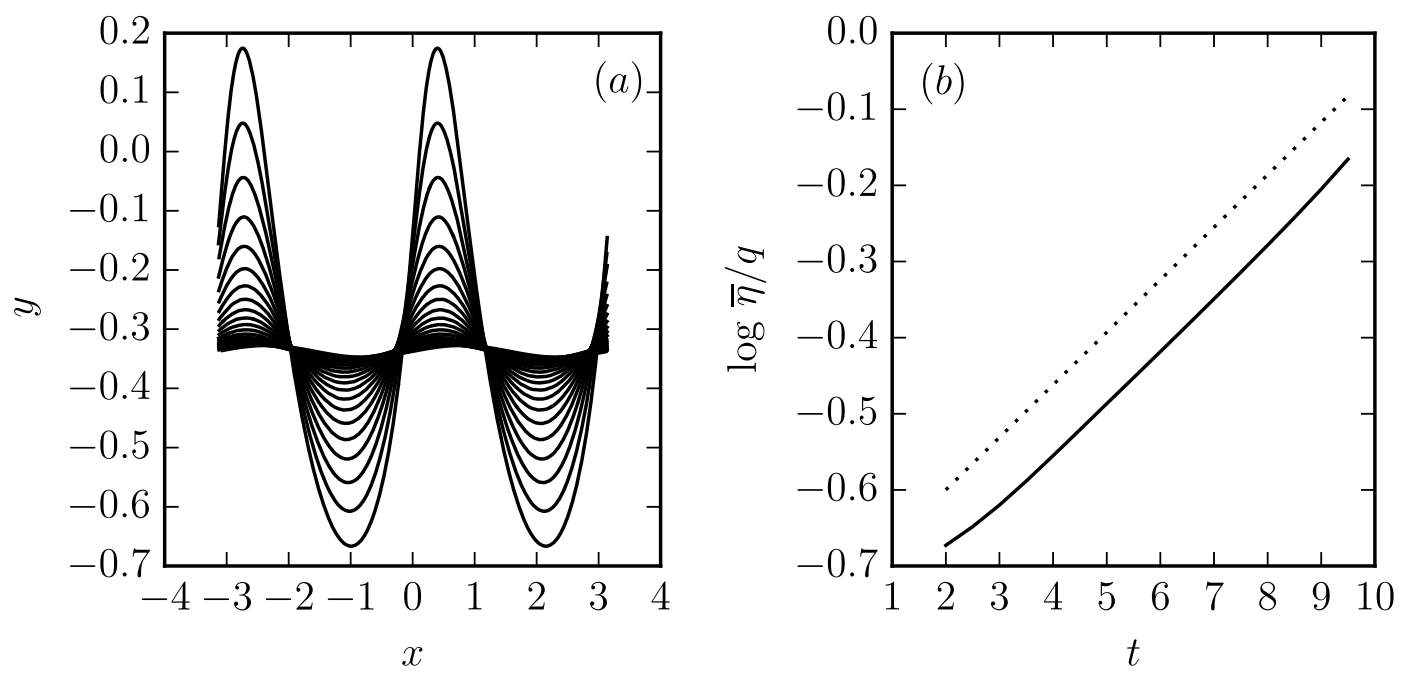

Figure 4. Growth of the perturbation: $\log (\bar{\ell})$ vs $t$ (black) and reference line of slope corresponding to the most amplified mode $\sigma_{i} / q=0.069$ (dotted line) for the filament forced by the most amplified mode. 

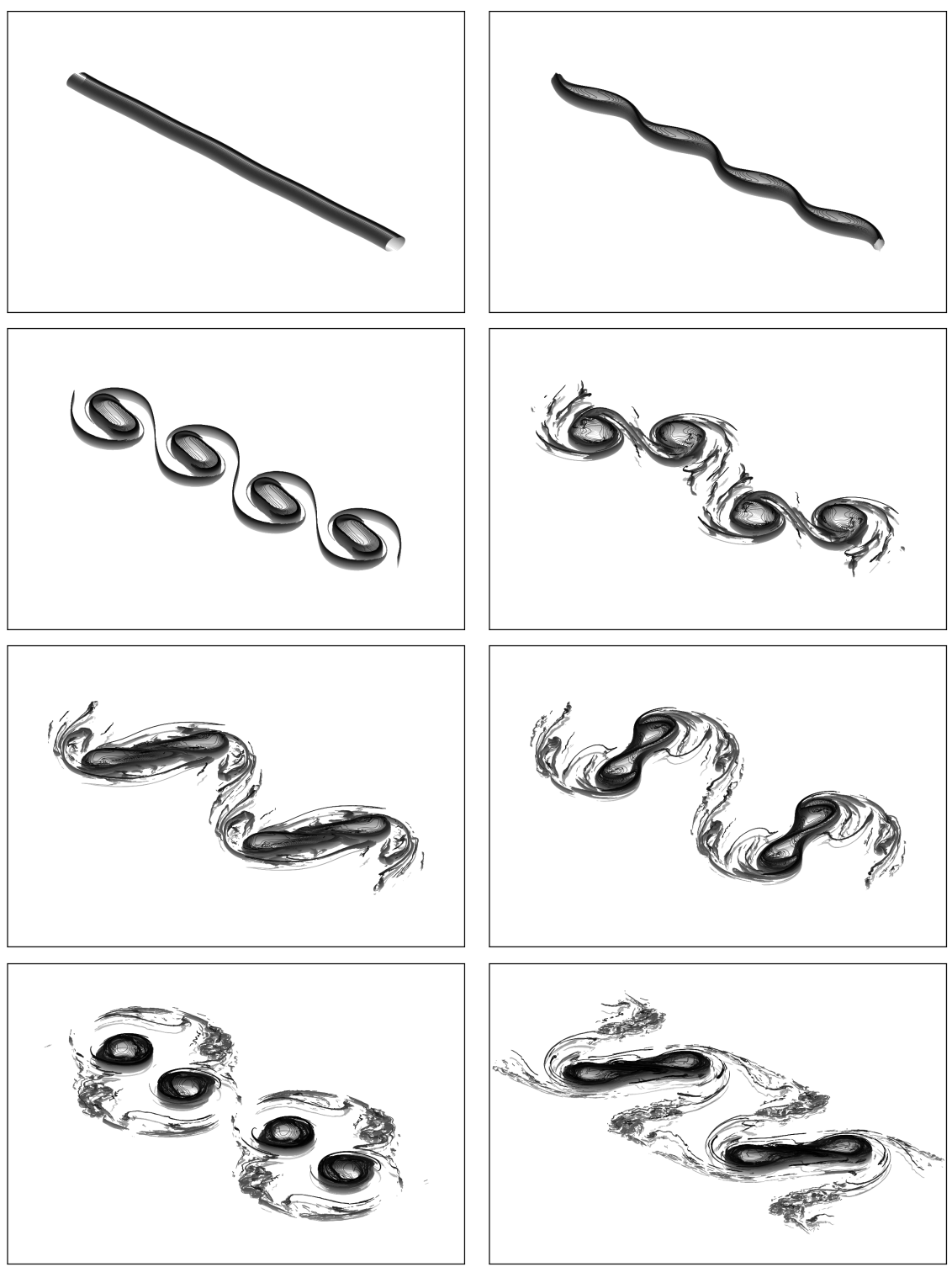

Figure 5. Evolution of a single filament of uniform PV $q=2 \pi$ of radius $a=0.169$, initially perturbed by the most amplified mode of nondimensional wavenumber $(k a)^{\max }=0.676$ and its first subharmonic $k a=0.338$ at $t=0,7,14,30,35$, 90.5 52.5 and 72.5. Colour of the contours indicates the height of the contour, with darker contours are nearer the top.

turbing both the most amplified mode with $(k a)^{\max }=0.676$ and its first subharmonic $(k a)^{\mathrm{sub}}=0.338$ with equal amplitude $\epsilon=0.01$. The radius of the filament is set to $a=0.169$ such that computational box contains two periods the subharmonic mode. Results are presented in figure 5 . As expected the most amplified mode growth faster than the subharmonic and four billows form. Then, due to the simultaneous growth of the first subharmonic, billows merge two by two to form billows of approximatively twice the size of the first generation of billows. These two secondary billows are however unstable and break back into four billows before merging again. This sequence of merger/break appear to repeat quasi-periodically. This is in contrast with the merger of the first generation of billows of the planar mixing layer where the secondary large billows are persistent (see Moser and Rogers 1993).

In the two previous numerical experiments the filament was initially perturbed by either a monochromatic wave or a wave and its first subharmonic, in phase. To break the symmetry of the perturbation, we next perturb the filament by a more complex wave. As before, the 
Table 1. Growth rates for the modes forced in $F_{m}$ defined in (19)

\begin{tabular}{ccc}
\hline$k$ & $k a$ & $\sigma_{i} / q$ \\
\hline 0.5 & 0.0845 & 0.0062 \\
1 & 0.169 & 0.017 \\
2 & 0.338 & 0.042 \\
3 & 0.507 & 0.061 \\
4 & 0.676 & 0.069 \\
5 & 0.845 & 0.059 \\
6 & 1.014 & 0.022 \\
7 & 1.183 & 0 \\
8 & 1.352 & 0 \\
\hline
\end{tabular}

perturbation consists of an initial deformation of the filament's boundary. The filament's radius is set to $a=0.169$. In this third numerical experiment, the deformation is given by

$$
F_{m}=\epsilon\left[\sin \left(\frac{y}{2}+\phi_{0}\right)+\sum_{k=1}^{8} \sin \left(k y+\phi_{k}\right)\right]+\epsilon^{\prime} \xi_{j}^{i}
$$

where $\epsilon=0.01, \epsilon^{\prime}=0.3 \epsilon,\left(\phi_{k}\right)_{0 \leq k \leq 8}$ are random phases for each monochromatic perturbation, and $\xi_{j}^{i}$ is an additional pseudo-random perturbation for each node $i$ along the contour $j$ with $0<\xi_{j}^{i}<1$, obtained by the pseudo-random number generating Fortran routine rand(). The growth rates of the modes are listed in table 1 . It should be noted that the last two modes for $k=7,8$ are neutrally stable but still provide some small amplitude initial deformation. Snapshots of the flow evolution are presented in figure 6. Initially five billows start to form in an asymmetric way due to the simultaneous growth of several perturbations. Two of the billows merge as they form and by the time the billow detach, there are four identifiable main structure. The largest one is formed by the merger of the two 'embryos' of billows mentioned above. This elongated billow is unstable and breaks asymmetrically by ejecting a smaller vortex. The later evolution is complex as neighbouring billows can temporarily merge and the merged structures break generating filaments and secondary smaller scale vortices. Near the end of the simulation, the evolution has settled and the flow consists mainly of three large billows which are roughly in a row, and two smaller satellite vortices.

To better describe the evolution of the flow, we identify the largest billow present in the flow, after its formation, and we analyse the time evolution of its volume. Results are presented in figure $7(a)-(c)$. For the first case investigated, with $a=0.338$ and a monochromatic forcing at $k a=(k a)^{\max }=0.676$, the billows are very stable after their formation and their volume is almost time invariant, apart from a very small loss of material to filaments and debris. The loss of volume between $t=17$ and $t=100$ is only about $2.6 \%$ for the largest billow and the tendency to shed material is decreasing with time, see figure $7(a)$. Figure $7(e)-(f)$ show the evolution of the best fitted ellipsoid semi-axis lengths. The best fitted ellipsoid for a given vortex of volume $V$ is the ellipsoid of centre $\boldsymbol{X}_{c}=(1 / V) \iiint_{\text {vortex }} \boldsymbol{x} \mathrm{d}^{3} \boldsymbol{x}$ and having the same second order geometrical moments $I_{i j}=\iiint_{v o r t e x}\left(x_{i}-X_{i}\right)\left(x_{j}-X_{j}\right) \mathrm{d}^{3} \boldsymbol{x}$ where $\left(x_{i}\right)_{\{i=1,2,3\}}=(x, y, z)$. The ellipsoid semi-axis lengths are then the eigenvalues of the $3 \times 3$ symmetric matrix whose coefficients are $B_{i j}=(5 / V) I_{i j}$. By convention, the three semi-axis lengths are ordered $\alpha \leq \beta \leq \gamma$. In all cases, the billows are flat and nearly upright-standing such that $\alpha$ is roughly half the vertical extend of the billows and is nearly time-independent in our experiments. Figure $7(d)$ shows the evolution of $(\alpha, \beta, \gamma)$ for the first case. We see that the billow pulsates periodically 'horizontally' as $\beta$ and $\gamma$ are nearly horizontal lengths. The oscillations of $\beta$ and $\gamma$ are in anti-phase. This is expected as the nearly conserved volume of the billow is $4 \pi \alpha \beta \gamma / 3$.

In the case of the filament of radius $a=0.169$ forced by the bi-chromatic wave with $k a=$ $(k a)^{\max }=0.676$ and $k a=(k a)^{\mathrm{sub}}=0.338$, the evolution of the volume of the largest billow 

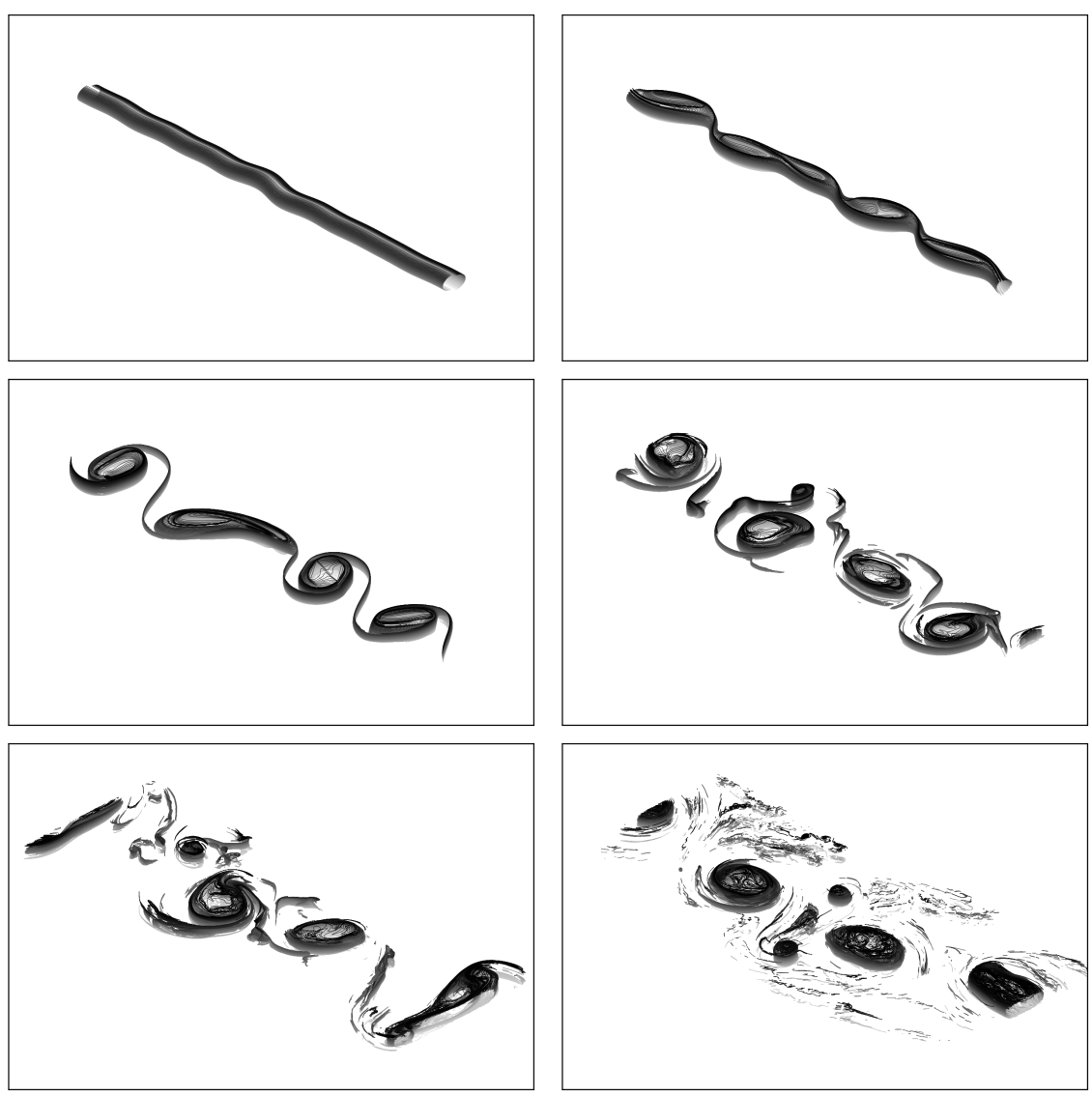

Figure 6. Evolution of a filament of uniform PV $q=2 \pi$ and of radius $a=0.169$ forced initially by the wave $F_{m}$ defined in (19). Snapshot of the bounding contours displayed at $t=0,6,10,20,40$ and 100.

shown in figure $7(b)$ clearly illustrates the quasi-periodic, temporary merger of neighbouring billows. Each merger forms a larger billow with is twice the size of the first generation of billows. As mentioned, this larger secondary billows are not stable. Figure 7(e) shows that the main axis has a length $\gamma^{6}$ which typically more than doubles during the merger process. The horizontal aspect ratio of the merged billow can be estimated by $\gamma / \beta$ and is typically larger than 2. The merged billow, submitted to the shear induced by the other billow, is therefore very deformed which explains why it eventually breaks.

Finally, figures $7(c)$ and $(f)$ show the evolution of the volume and the semi-axis lengths for the filament of radius $a=0.169$ and the multi-frequency forcing $F_{m}$. Here is situation is more complex with different billows merging and breaking. As mentioned before, the system eventually settles with three well-separated main billows present in the flow.

\section{Jet}

Formally, the procedure described in the section 3 to address the linear stability of the filament can be straightforwardly adapted to multiple parallel filaments, or to filaments with distribute PV. Indeed, by linearity of the inversion relation (2), the basic state solution can be constructed by adding the elementary solution for each individual filaments. Additionally, the integral in (18) remains formally the same as far as the integration is performed on all filaments and all PV jumps. We therefore extend our investigation to two other classical configurations. In the first one, we construct a jet-like flow by placing two filaments of uniform 

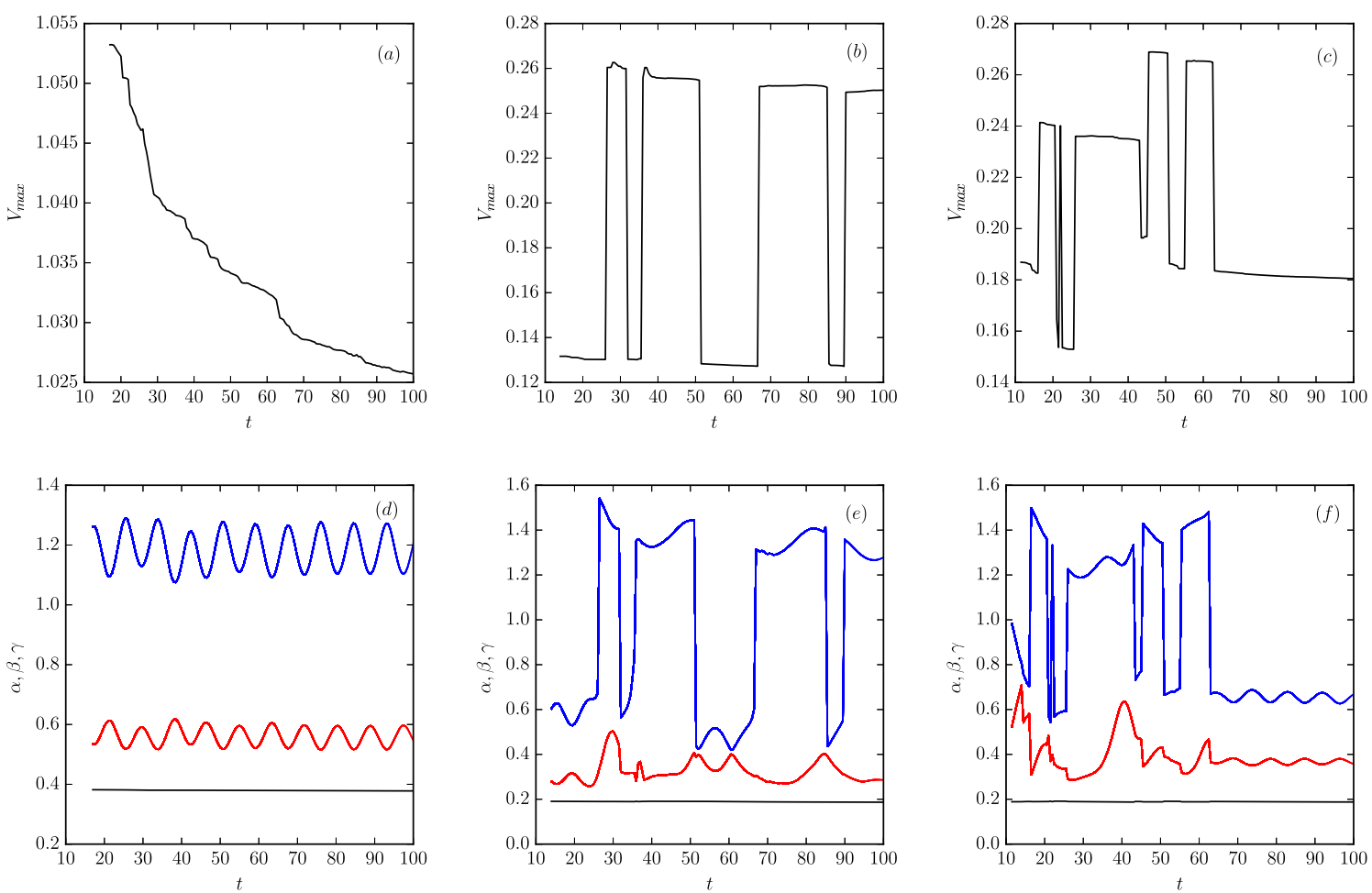

Figure 7. $(a)-(c)$ Evolution of the volume of the largest billow generated by the roll-up of a single filament of radius $a$ and of PV $q=2 \pi$ and for $(a): a=0.338$ and a monochromatic forcing with $k a=(k a)^{\max }=0.676,(b) a=0.169$ and a bi-chromatic forcing with $k a=(k a)^{\max }=0.676$ and $k a=(k a)^{\text {sub }}=0.338$ and $(c): a=0.169$ and the forcing $F_{m}$ defined in $(19)$. $(d)-(f)$ Evolution of the best fitted ellipsoid semi-axis lengths for $(d)$ the same case as $(a)$, then $(e)$ same as $(b)$ and $(f)$ same as $(c)$.

opposite PV and of equal radius $a$ next to each other. The axes of both filaments are parallel to the $x$-axis and are located in the plane $z=0$ at $y=-a$ and $y=a$ respectively. Profiles of longitudinal velocity are presented in figure 8. It should be noted that whether the filament of positive PV is located to the right or to the left of the negative PV filament is dynamically irrelevant. This only changes the sign of the velocity field. The evolution of the two configurations is perfectly symmetric. Since the velocity field is height-dependent, there is a vertical shear. This shear is antisymmetric with respect to the plane $z=0$ since the basic state velocities are symmetric $\bar{u}(y, z)=\bar{u}(y,-z)$.

Figure 9 shows the growth rate of the unstable modes. Growth rates are calculated for $k a \in[0.01,1.1]$, every $\Delta(k a)=0.001$. Two modes of instability are identified. They differ from the symmetry of the deformation. The most amplified mode is the sinuous mode for which the deformation of both filaments is identical. The second mode is the varicose mode for which the deformation of the filaments is symmetric with respect to the vertical plane $(y=0)$ separating the two filaments. Both modes have a vanishing growth rate $\sigma_{i}$ for infinitely long waves $k a \rightarrow 0$. The dominant (sinuous) mode is unstable to perturbation whose nondimensional wavenumber $(k a)_{s} \in(0,1.188)$. The most amplified mode corresponds to $(k a)_{s}^{\max }=0.768$ and has a nondimensional growth rate $\sigma_{s}^{\max } / q \simeq 0.1007$. The varicose mode is unstable to perturbations whose nondimensional wavenumber $(k a)_{v} \in(0,0.734)$ and the maximum growth rate is $\sigma_{v}^{\max } / q=0.0162$ for $(k a)_{v}^{\max }=0.419$.

We illustrate the evolution of the jet initially perturbed by the mode of nondimensional wavenumber $k a=0.8$ for which $\sigma_{i} / q \simeq 0.1003$, which is close the most unstable mode. The rods radius is set to $a=0.4$, and the mode $k=2$ is forced initially by deforming both filaments by a wave $\epsilon \sin 2 x$, with $\epsilon=0.01$. Results are presented in figure 10 . The jet breaks into a 


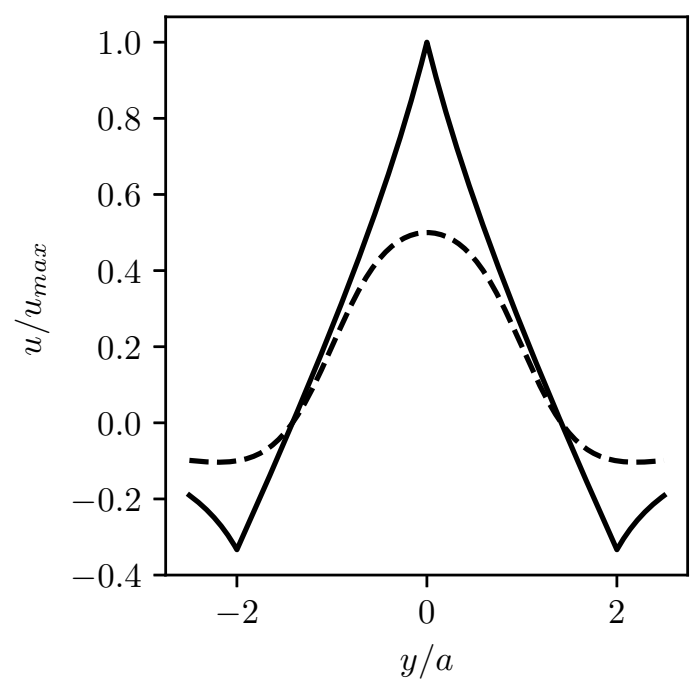

Figure 8. Velocity profile for the jet formed by two filaments aligned in the $x$-direction in the plane $z=0$ (solid line) and $z=a$ (dashed line).

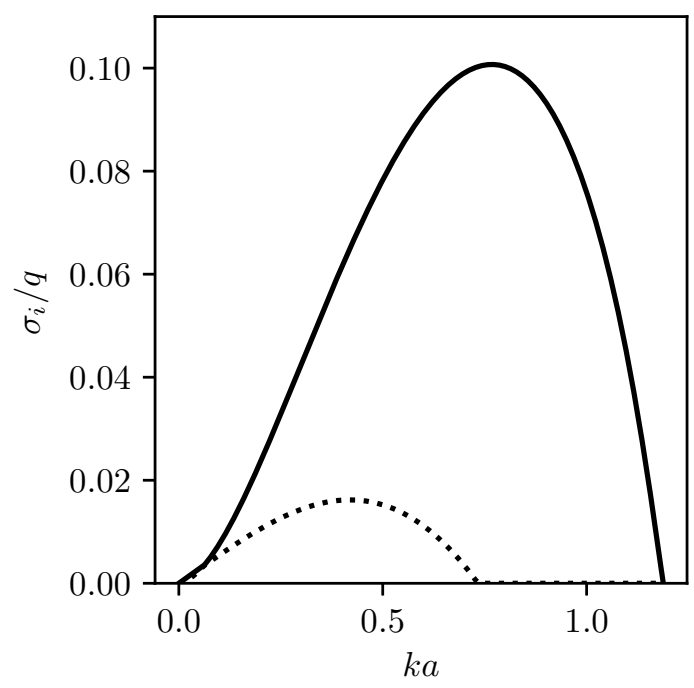

Figure 9. Growths rate $\sigma_{i}$ of the two unstable modes for a jet rescaled by the filament's PV $q$ vs the nondimensional longitudinal wave number $k a$.

street of alternate sign billows connected by thinning jets formed by tongues of opposite sign PV. As the billows form and roll, the apparent width of the jet increases in time. Figure 11 shows the time evolution of $\Delta=y_{\max }-y_{\min }$ the distance between the two outer horizontal edges of the 'temporal' jet in the transversal $y$-direction. After the initial (linear) growth of the perturbation, there is an almost linear increase in the jet's 'width' with time in the interval $t \in[6.10]$, followed by a saturation. The saturation of the increase of the width is due to the saturation of the instability, and due to the fact that the first subharmonic is not perturbed. It is also influenced by the fact that the billows start to become large compared to the size of the periodic domain and therefore the billows interact with their periodic images.

We do not illustrate the nonlinear evolution of the varicose mode as it is never the dominant mode. 

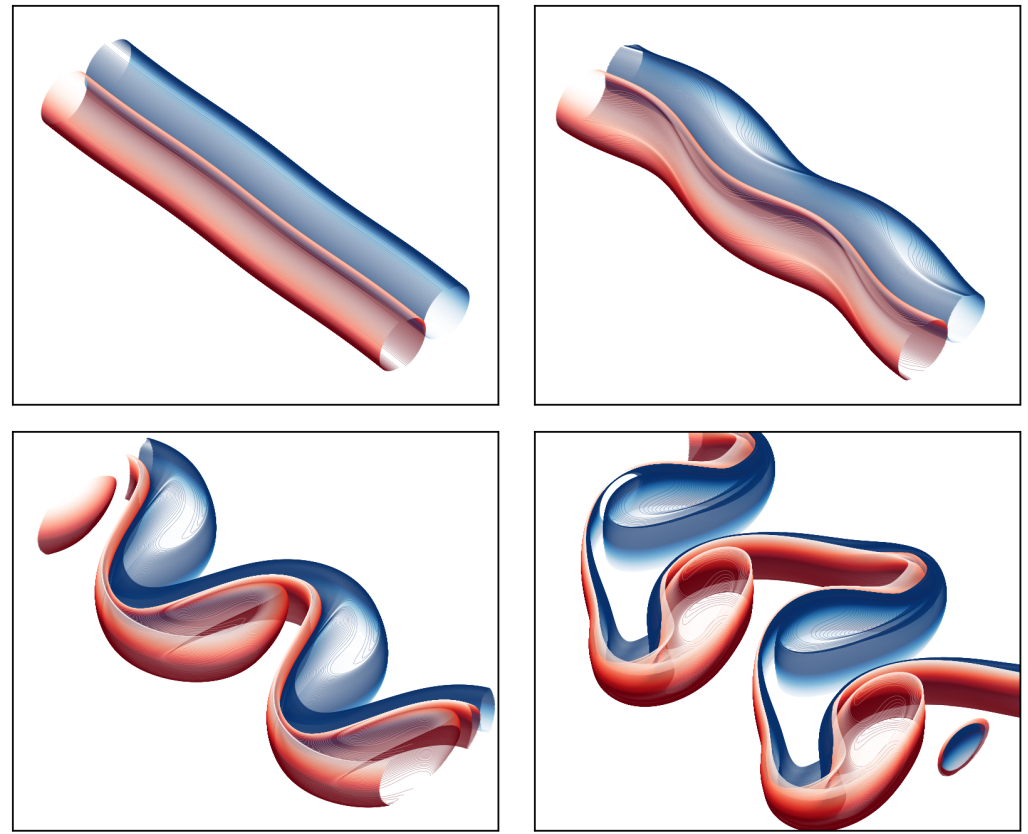

Figure 10. Evolution of a jet formed by two filaments of uniform PV $q= \pm 2 \pi$ of axis $y= \pm a$ and radius $a$. Snapshots on the bounding contours displayed at $t=0,4,7$ and 10. Colour saturation indicates height with darker contours near the bottom. Blue contours bound negative PV while red contours bound positive PV. (Colour online)

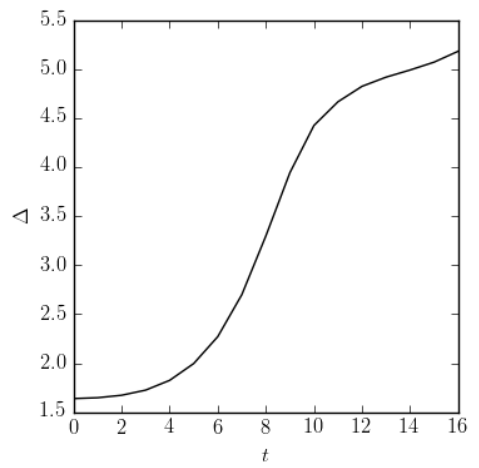

Figure 11. Maximum width of the jet formed by two filaments of radius $a=0.4$ vs time.

\section{Hetonic filaments}

\subsection{Pure baroclinic case}

Finally we consider the flow generated by two filaments of uniform, opposite PV, $q= \pm 2 \pi$, and of radius $a$ whereaxes of the filaments are in the plane $y=0$ and located at $z=-a$ and $z=a$ respectively. PV therefore changes sign in the vertical direction. By symmetry, whether the filament with positive PV is on the top or bottom is dynamically irrelevant. This only changes the sign of the velocity field. The velocity profile for the basic state is presented in figure 12 in two cross-sections on the upper half domain $z>0$, assuming positive PV for the upper filament. By symmetry the velocity is the same but opposite in sign in the lower domain $z<0$. Finally, due to symmetry again, $(u, v)=(0,0)$ in the plane $z=0$. This configuration induces a strong vertical shear.

Using the same formalism as for the single filament and the jet, we investigate the linear 


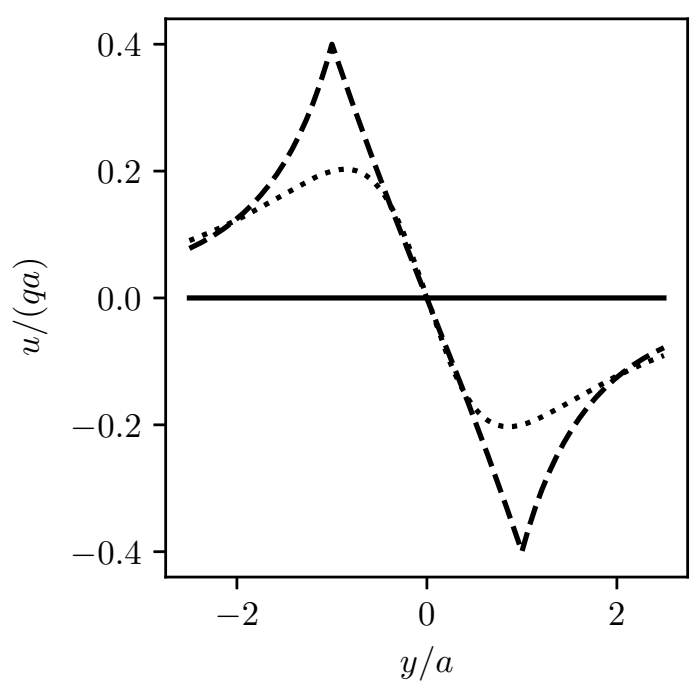

Figure 12. Velocity profile for the hetonic filaments formed by two filaments aligned in the $x$-direction in the plane $y=0$ (solid line), $z=a$ (dashed line) and $z=2 a$ (dotted line).
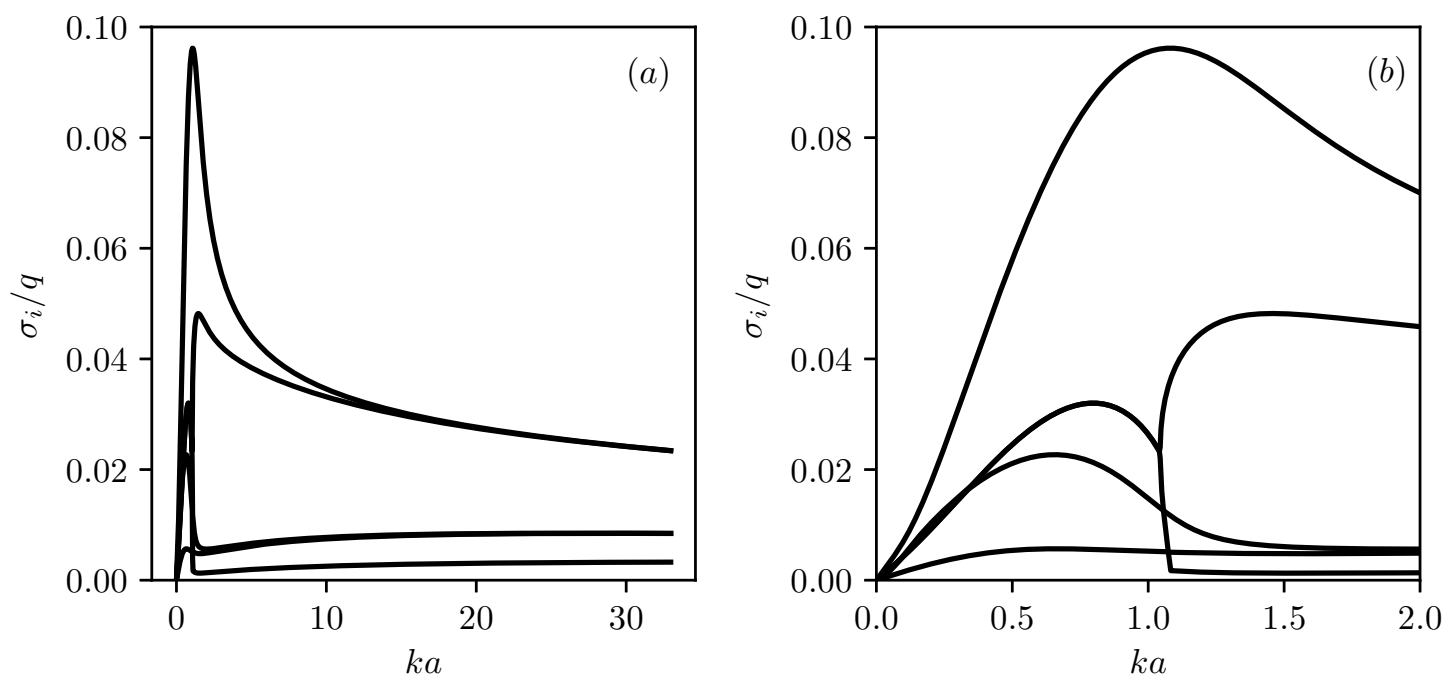

Figure 13. (a) Growth rates $\sigma_{i}$ of the five most amplified modes rescaled by the filaments PV magnitude $q$, for the hetonic filaments centred on the axes $\pm(0, a)$ vs the nondimensional wavenumber $k a$. $(b)$ same as $(a)$ but close up on the range $k a \in[0,2]$.

stability of the hetonic filaments. Due to the extended range of $k a$ where instable modes are found, growth rates are first calculated in the interval $k a \in[0.03,3.3]$ every $\Delta(k a)=$ 0.003 , then in the interval $k a \in[3.3,33)$ every $\Delta(k a)=0.03$. Results are shown in figure 13 . The situation is fundamentally different from the previous cases. Although all modes have a vanishing growth rate $\sigma_{i} \rightarrow 0$ as $k \rightarrow 0$, which is consistent with (18), there is no overall large wavenumber cut-off. The most amplified mode reaches a maximum growth rate $\sigma_{i} / q \simeq 0.0962$ for $(k a)_{h}^{\max }=1.08$. The growth rate does not however collapse to 0 for some finite $(k a)_{c}$ but slowly decreases for $k a$ is increased.

We first illustrate the nonlinear evolution of the hetonic rods for $r=0.4$ with a monochromatic forcing by the wave $\epsilon \sin 2 x, \epsilon=0.01$ as in the previous numerical experiments. This corresponds to $(k a)=0.8$. The linear theory predicts $\sigma_{i} / q=0.0874$, which is close to the 

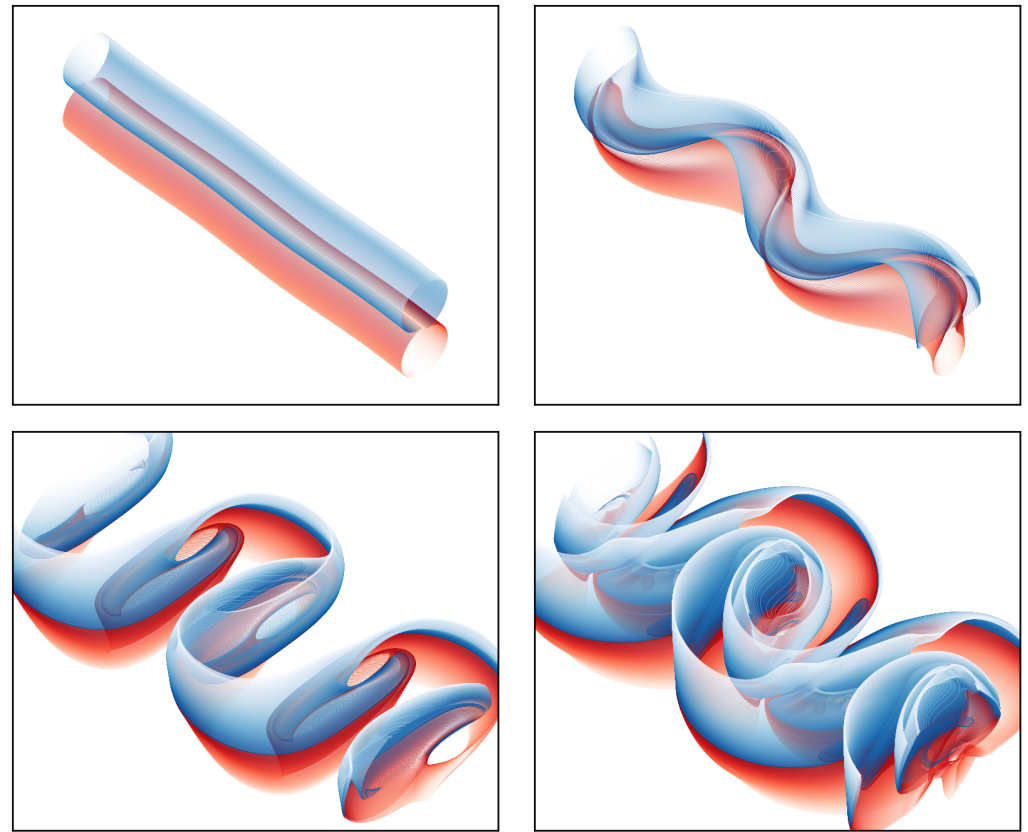

Figure 14. Evolution of hetonic filaments of PV $q= \pm 2 \pi$ centred on the axes $\pm(0, a)$. The filaments have radius $a=0.4$ and are forced by the wave $\propto \sin 2 x(k a=0.8)$. Snapshots on the filaments bounding contours are displayed at $t=0,7,12,17$. Colour saturation indicates height with darker contours near the plane $z=0$. Blue contours bound negative PV while red contours bound positive PV. (Colour online)
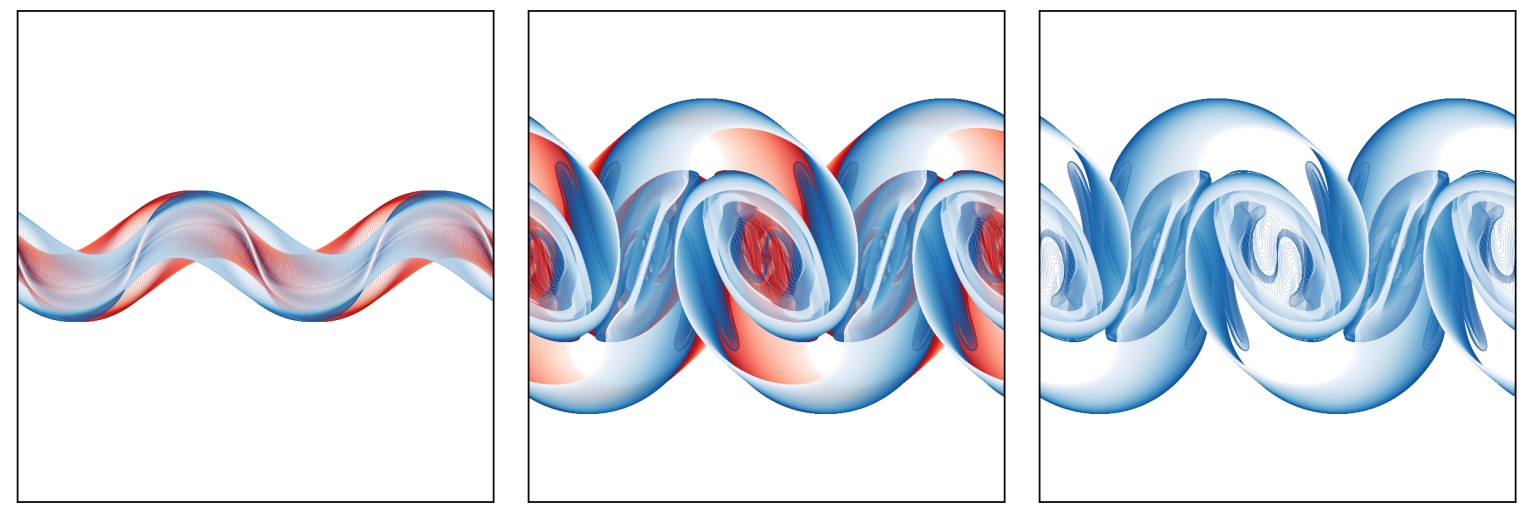

Figure 15. Same as figure 14 but top view on the filaments bounding contours at $t=7$ (left) and 17 (centre and right). The panel on the right is the same as the panel in the middle but we mask the lower filament to better reveal the details of the deformation of one filament. (Colour online)

most amplified mode. Results are shown in figures 14 and 15.

The filaments break baroclinically, with billows forming in both the upper and lower filaments with a phase of $\pi / 2$. The evolution becomes rapidly complex. The billows formed by the roll-up of the lower filament (respectively upper filament) induces a local rotation of the tongues of PV joining the billows formed in the upper filament (respectively lower filaments). It should be that this local rotation of the tongues of PV is forced by the other filament, hence the orientation of the rotation is opposite the direction of the shear in the PV tongue. This local rotation is naturally more important there the filaments are at their nearest and is not uniform in the vertical direction across the PV tongues connecting the main billows. It should be noticed that throughout the time evolution, the entire filaments are affected by the instability. 

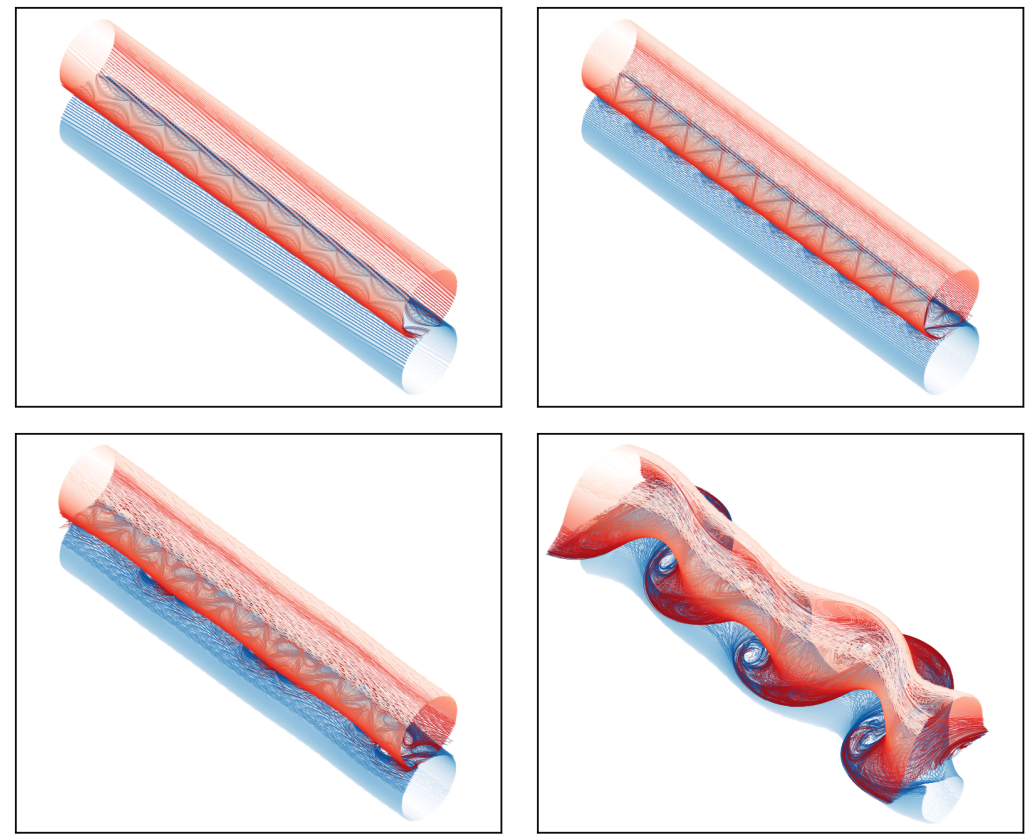

Figure 16. Evolution of hetonic filaments of PV $q= \pm 2 \pi$. The filaments have radius $a=0.5$ and are forced by the wave $\propto \sin 10 x(k a=5)$. Snapshots on the filaments bounding contours are displayed at $t=8,13,24,29$. Colour saturation indicates height with darker contours near the plane $z=0$. Blue contours bound negative PV while red contours bound positive PV. (Colour online)
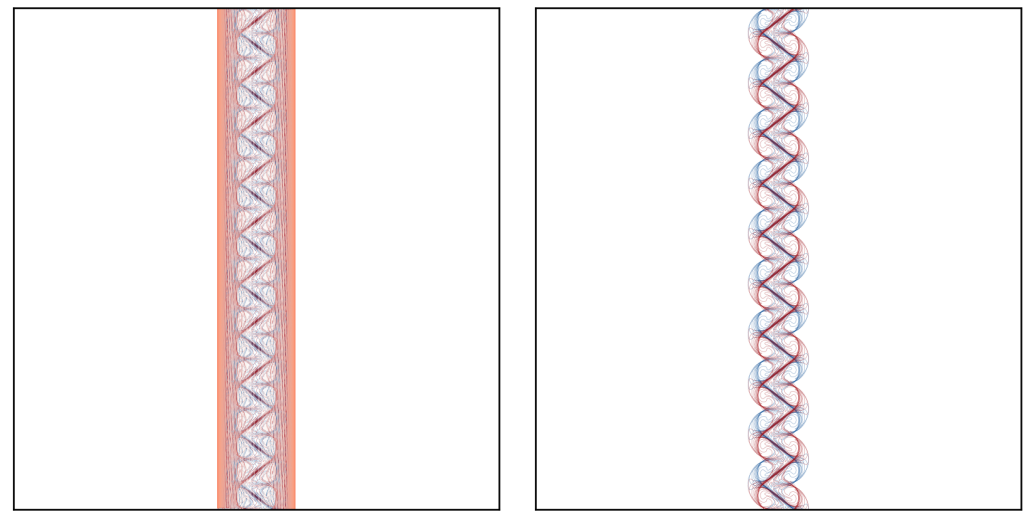

Figure 17. Same as figure 16 but top view on the filaments bounding contours at $t=13$ (right panel). The left panel shows the top view over the filaments bounding contours in the central region $|z|<0.067 a$. (Colour online)

We finally illustrate the evolution of the hetonic filaments when forced by the high wavenumber wave. We set $a=0.5$ and we force the filament by the wave $\epsilon \sin 10 x, \epsilon=0.01$. The growth rate of the most amplified mode of normalised wavelength $k a=5$ is $\sigma_{i} / q=0.044$. Recall that both the single filament or the jet were neutrally stable to perturbation of such high nondimensional wavenumber. The evolution of the hetonic filaments is presented in figures 16 and 17. We see that the perturbation indeed grows. But the instability first grows locally, in region near the plane $z=0$ where the two filaments touch and PV changes sign. Eventually, the nonlinear evolution of the high wavenumber instability excites modes at lower wavenumber. Three large billows form on each filament, indicating the growth of the mode $k a \simeq 1.5$ among other modes. The growth of these larger wavelength deformations, which have a larger growth rate, spreads to the full filaments, and eventually breaks them by re-organising the filaments into billows. 


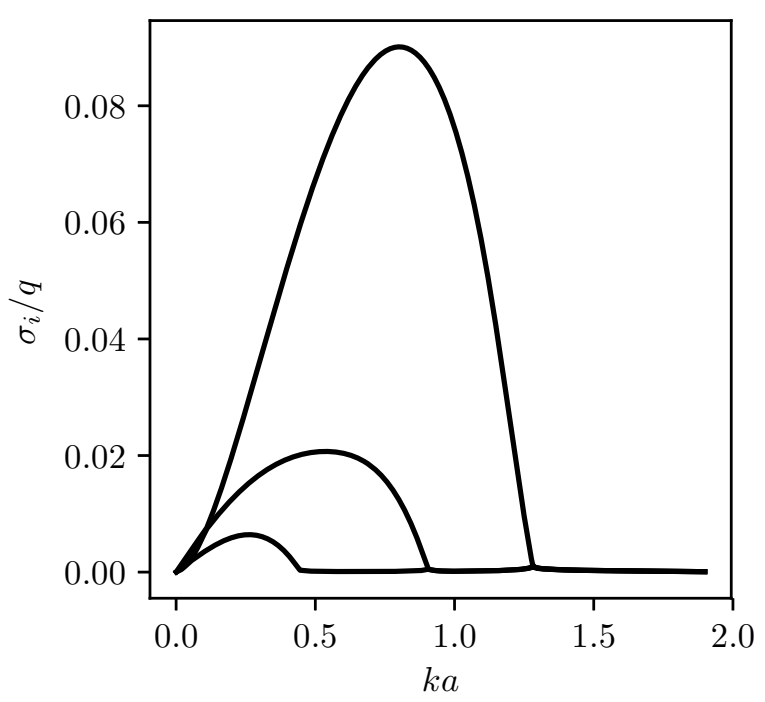

Figure 18. Growth rates $\sigma_{i}$ of the five most amplified modes rescaled by the filaments PV magnitude $q$, for the hetonic filaments centred on the axes $\pm(a / \sqrt{2}, a / \sqrt{2})$ vs the nondimensional wavenumber $k a$.

\subsection{Intermediate vertical offset}

The final example corresponds to a situation where PV changes sign both in the horizontal (allowing for barotropic modes) and vertically (allowing for baroclinic modes). As in the previous case the two filaments have the same radius $a$ and are centred along the axes $(y, z)=$ $\pm(a / \sqrt{2}, a / \sqrt{2})$. We call filament 1 the one along $(a / \sqrt{2}, a / \sqrt{2})$, and filament 2 the other one. The growth rate of the three most unstable modes are plotted in figure 18 for $n=4000$. No other mode is observed apart for some small, resolution dependent, numerical noise observed for large wavenumber modes. The maximum growth is $\sigma_{i} / q=0.0901$ for $(k a)^{\max }=0.802$. This mode is unstable in the range $k a \in(0,1.28)$. The two other modes have maxima for $\left(\sigma_{i} / q,(k a)^{\max }\right)=(0.0201,0.537)$ and $(0.0063,0.237)$ respectively. They are unstable in the ranges $(0,0.9)$ and $(0,0.45)$, respectively. The norm of the eigenvectors $\mid \eta(\theta)$ are given in figure 19. Recall that $\theta$ is the local angle from direction the axis $y=$ const. We next denote $\theta_{i}$ the local angle for filament $i, i=1,2$. For the first two modes, the maximum deformation is in the region where the two filaments are in contact $\left(\theta_{1}^{c} \sim 5 \pi / 4, \theta_{2}^{c} \sim \pi / 4\right)$ but on the face opposite: $\theta_{1}^{d}=\pi-\theta_{1}^{c}=-\pi / 4$ and $\theta_{2}^{d}=\pi-\theta_{2}^{c}=3 \pi / 4$ (see schematic drawing in figure 20). The third most amplified mode has maximum deformation at the bottom of lower filament 2 and top of upper filament 1.

We next conduct a numerical simulation of the configuration using CASL as in the previous case. We set $a=0.2$ for both filaments and we perturb the flow by deforming initially the two filaments by a sinewave with $k=4$, corresponding to $(k a)=0.8 \simeq(k a)^{\max }$, and of amplitude 0.01 . The flow evolution is presented in figure 21. The flow reorganises itself into a street of alternate sign billows, offset both vertically and horizontally, as well as series of smaller scale billows on the external sides of the main structures.

We also indicate the stability results $\sigma(k a)$ for parallel filaments of opposite PV whose axis are located at $(y, z)= \pm a(\cos \tau, \sin \tau)$ for $\tau=\pi / 8$ and $8 \pi / 3$ in figure 22 . As in the previous case, results are shown for $n=4000$. For the first case with $\tau=\pi / 8$, results are shown for $k a \in[0.001,1.9]$ every $\Delta(k a)=0.001$, and for the second case with $\tau=8 / p i$ for $k a \in[0.002,3.6]$ every $\Delta(k a)=0.002$ due to the extended range of values investigated. We see that the shape of the stability curves $\sigma(k a)$ is continuously changing from the case $\tau=0$ (the case of the jet presented in section 5) to the case $\tau=\pi / 2$ (the case of the pure 

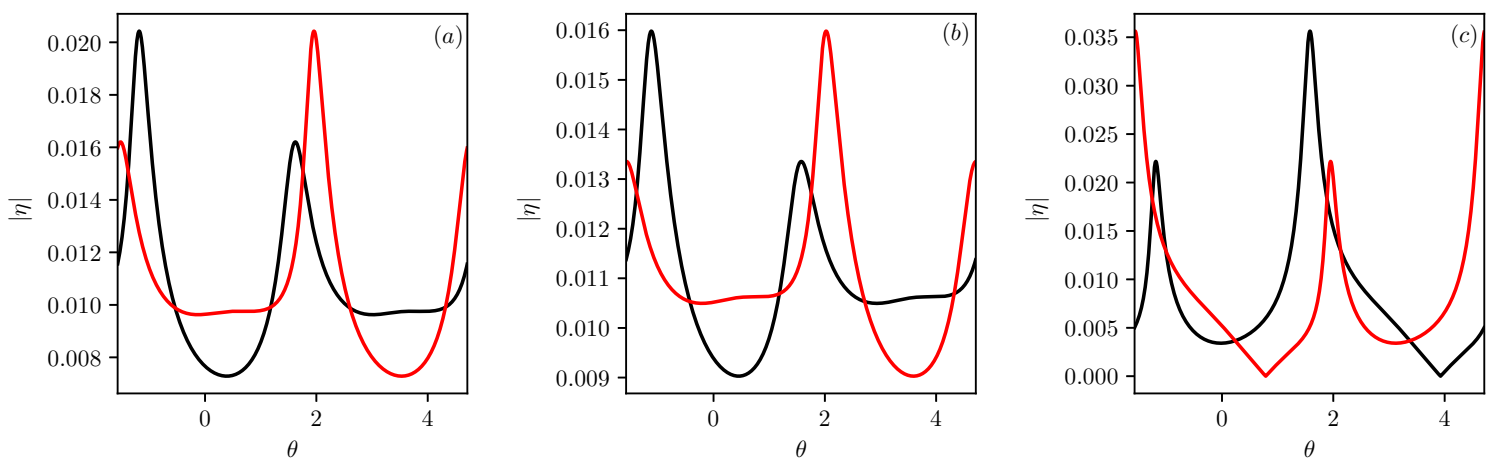

Figure 19. Norm of the eigenvector $|\eta|(\eta \in \mathrm{C})$ for $(a)$ the most amplified mode $\left(\sigma_{i} / q, k a\right)=(0.0901,0.802),(b)$ the most amplified secondary mode $\left(\sigma_{i} / q, k a\right)=(0.0201,0.537)$, and $(c)$ the most amplified tertiary mode $\left(\sigma_{i} / q, k a\right)=$ $(0.0063,0.237)$ vs the angle $\theta$. The black line corresponds to the filament aligned along the axis $(y, z)=(a / \sqrt{2}, a / \sqrt{2})$ and the red line corresponds to the filament aligned along the axes $(y, z)=(-a / \sqrt{2},-a / \sqrt{2})$.

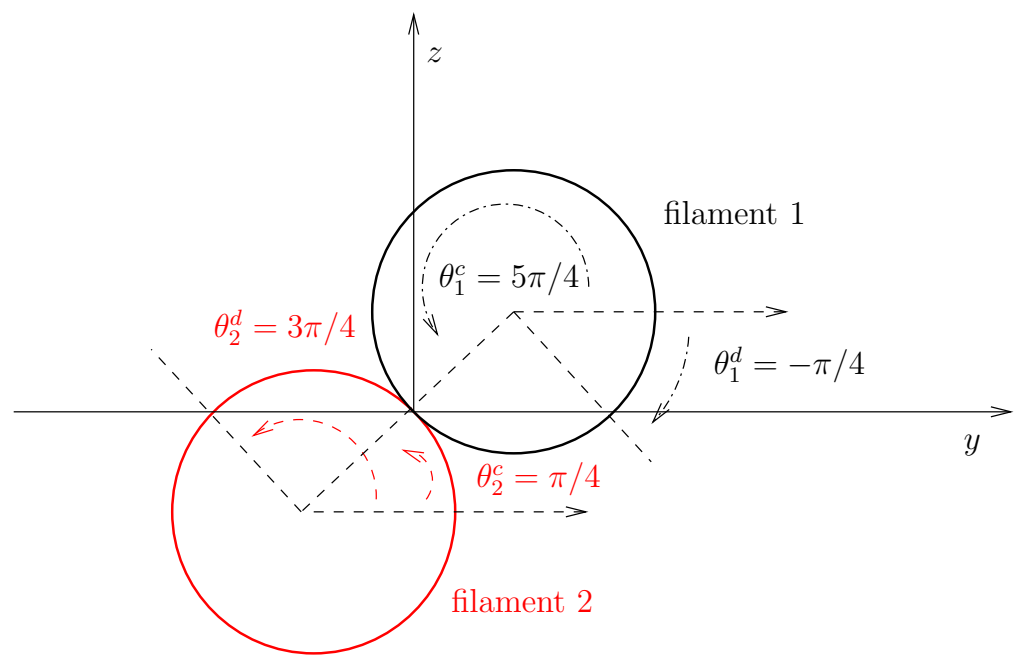

Figure 20. Schematic view of the location of the maximum deformation $\left(\theta_{i}^{d}\right)$ for the two most unstable modes for the pair of filaments aligned on the axes $\pm(a / \sqrt{2}, a / \sqrt{2})$.

baroclinic hetonic filaments investigated in section 6.1). In particular, we see the presence of a tail of instability $\sigma_{i}>0$ for large $k a$ if the angle $\tau$ is large enough, indicating the influence of baroclinic instability.

\section{Conclusion}

We have analysed for the first time the stability of filament of uniform quasi-geostrophic $\mathrm{PV}$. These filaments provide typical shear zones in a three-dimensional flow. The equations derived for the linear stability can be formally used to address the linear stability of any flow induced by an arbitrary number of parallel PV filaments and by filaments with distributed PV. The linear stability of a single filament shows that such shear zones are sensitive to Kelvin-Helmholtz instabilities for perturbations in a finite range of wavenumbers, similarly to the classical planar or two-dimensional vorticity strip. The most amplified mode has however much shorter wavelength.

We have extended results to a jet formed by the flow induced by two touching, parallel PV filaments whose axes lie in the same horizontal plane. As most jets, this jet is sensitive to two 

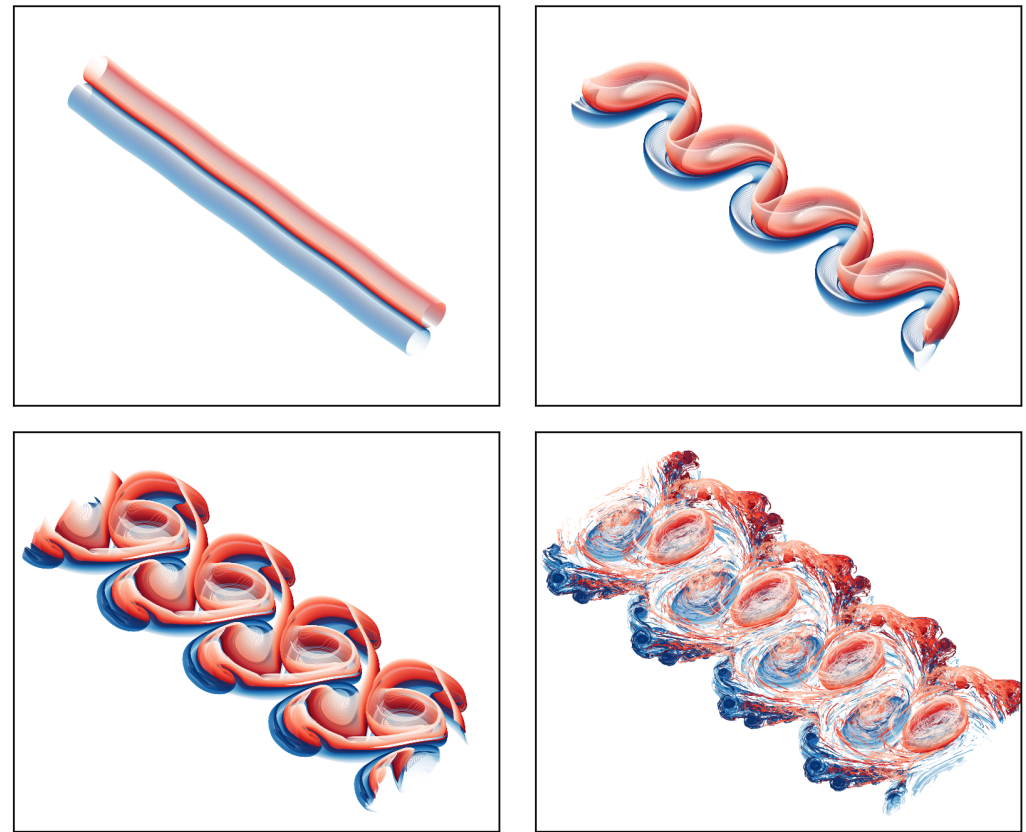

Figure 21. Evolution of the filaments of PV $q= \pm 2 \pi$ for an intermediate vertical offset. The filaments have radius $a=0.2$ and are forced by the wave $\propto \sin 4 x(k a=0.8)$. Snapshots on the filaments bounding contours are displayed at $t=0,8,15,71$. Colour saturation indicates height with darker contours near the plane $z=0$. Blue contours bound negative PV while red contours bound positive PV. (Colour online)
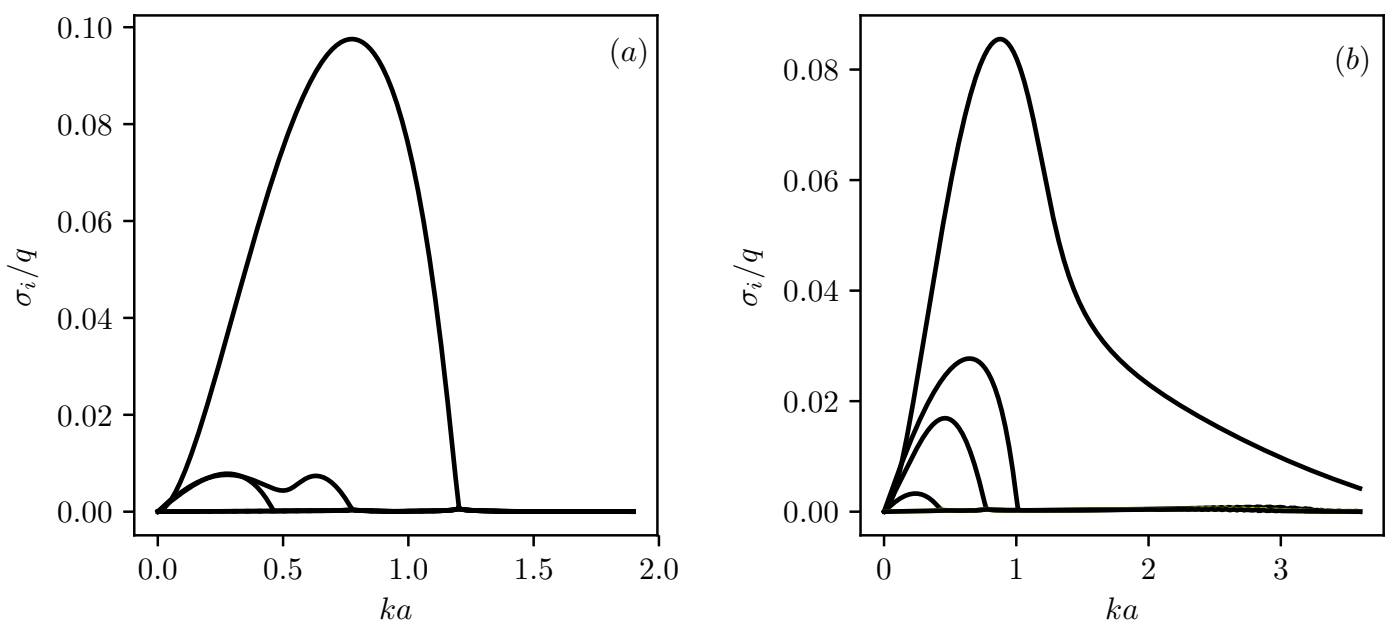

Figure 22. Growth rates $\sigma_{i}$ of the five most amplified modes rescaled by the filaments PV magnitude $q$, for the hetonic filaments centred on the axes $\pm a(\cos \tau, / \sin \tau)$ vs the nondimensional wavenumber $k a$ for $\tau=\pi / 8(a)$ and $\tau=3 \pi / 8(b)$

modes of instability which differ from their symmetry. The dominant, most amplified mode is the sinuous mode. The secondary, less amplified mode is the varicose mode. Both modes are unstable within a finite range of wavenumbers.

The situation changes if the filaments are placed on the top of one another. In this configuration, there is strong vertical shear and a change of sign of PV in the vertical direction. Such a configuration is sensitive to baroclinic unstable modes which exist over a large range of wavenumbers. The most amplified mode correspond to nondimensional wavenumbers $k a=\mathrm{O}(1)$. Higher wavenumber modes mainly first affect a thin region of the filaments, near 
the region where they touch. It can be noted that since the filaments have vertical circular cross-sections, one can always locally find a horizontal cross section of the filaments of arbitrary small width $\delta=2 \sqrt{a^{2}-z^{\prime 2}}$ provided $z^{\prime}$ is close enough to $a$, where $z^{\prime}$ is the vertical distance from the filaments axis. This means that, locally, $k \delta$ can be $\mathrm{O}(1)$ even for large values of $k$. This may explain the persistence of the mode of instability for high wavenumber perturbations and explain why the instability first develops locally.

The analysis of the stability of a single filament can shed some light on the stability of threedimensional filaments in a QG flows. Filaments are ubiquitous in QG dynamics. They can are formed, for example, by the stretching and shearing of small vortices and also during most vortex interactions. These filaments are therefore also typically evolving under the influence of external strain. The stability and the behaviour of three-dimensional PV filaments in a straining flow should be addressed in a future work.

\section{References}

Abramowitz, M. and Stegun, I.A., Handbook of Mathematical Functions with Formulas, Graphs, and Mathematical Tables, Tenth printing edn, 1972 (Washington, DC, USA: U.S. Government Printing Office).

Bambrey, R.R., Reinaud, J.N. and Dritschel, D.G., Strong interactions between two co-rotating quasigeostrophic vortices. J. Fluid Mech., 2007, 592, 117-133.

Dritschel, D.G., Contour surgery: a topological reconnection scheme for extended integrations using contour dynamics. J. Comput. Phys., 1988, 77, 240-266.

Dritschel, D.G., An exact steadily rotating surface quasi-geostrophic elliptical vortex. Geophys. Astrophys. Fluid Dyn., 2011, 105, 368-376.

Dritschel, D.G. and Ambaum, M.H.P., A contour-advective semi-Lagrangian numerical algorithm for simulating fine-scale conservative dynamical fields. Quart. J. Roy. Meteorol. Soc., 1997, 123, 1097-1130.

Dritschel, D.G. and Polvani, L., The roll-up of vorticity strips on the surface of a sphere. J. Fluid Mech., 1992, 234, 47-69.

Dritschel, D.G., Scott, R.K. and Reinaud, J.N., The stability of quasi-geostrophic ellipsoidal vortices. J. Fluid Mech., 2005, 536, 401-421.

Dritschel, D.G. and Viúdez, A., A balanced approach to modelling rotating stably stratified geophysical flows. J. Fluid Mech., 2003, 488, 123-150.

Fontane, J., Joly, L. and Reinaud, J., Fractal Kelvin-Helmholtz breakups. Phys. Fluids, 2008, 20, 091109.

Helmholtz, H., Monatsbericht. Konigl. Akad. Wiss. Berlin, 1868, pp. 215-228.

Juckes, M., Instability of surface and upper-tropospheric shear lines. J. Atmos. Sci., 1995, 52, 3247-3262.

Mashayek, A. and Peltier, W.R., Shear-induced mixing in geophysical flows: does the route to turbulence matter to its efficiency?. J. Fluid Mech., 2013, 725, 216-261.

McWilliams, J.C., The vortices of geostrophic turbulence. J. Fluid Mech., 1990, 219, 387-404.

Michalke, A., On the inviscid instability of the hyperbolic-tangent velocity profile. J. Fluid Mech., 1964, 19, $543-556$.

Moser, R.D. and Rogers, M.M., The three-dimensional evolution of a plane mixing layer: pairing and transition to turbulence. J. Fluid Mech., 1993, 247, 275-320.

Rayleigh, Lord., The theory of sound, Vol. II, 2nd edn, 1896 (New York: Dover publications).

Reinaud, J., Joly, L. and Chassaing, P., The baroclinic secondary instability of the two-dimensional shear layer. Phys. Fluids, 2000, 12, 2489-2505.

Reinaud, J.N. and Carton, X., The stability and the nonlinear evolution of quasi-geostrophic hetons. J. Fluid Mech., 2009, 636, 109,135.

Reinaud, J.N. and Dritschel, D.G., The critical merger distance between two co-rotating quasi-geostrophic vortices. J. Fluid Mech., 2005, 522, 357-381.

Reinaud, J.N., Dritschel, D.G. and Carton, X., Interaction between a surface quasi-geotrophic buoyancy filament and an internal vortex. Geophys. Astrophys. Fluid Dyn., 2016, 110, 461-490.

Reinaud, J.N., Dritschel, D.G. and Koudella, C.R., The shape of vortices in quasi-geostrophic turbulence. J. Fluid Mech., 2003, 474, 175-191.

Scott, R.K., A scenario for finite-time singularity in the quasigeostorphic model. J. Fluid Mech., 2011, 687, $492-502$.

Staquet, C., Two-dimensional secondary instabilities in a strongly stratified shear layer. J. Fluid Mech., 1995, 296, 73-126.

Waugh, D.W. and Dritschel, D.G., The stability of filamentary vorticity in two-dimensional geophysical vortexdynamics models. J. Fluid Mech., 1991, 231, 575-598. 